\title{
Evaluation of a novel, ultrathin, tip-bending endoscope in a synthetic force-sensing pancreas with comparison to medical guide wires
}

This article was published in the following Dove Press journal:

Medical Devices: Evidence and Research

22 December 2011

Number of times this article has been viewed

\author{
John E Chandler' \\ Cameron M Lee' \\ Alexander P Babchanik' \\ C David Melville' \\ Michael D Saunders ${ }^{2}$ \\ Eric J Seibel' \\ 'Human Photonics Laboratory, \\ Department of Mechanical \\ Engineering, University of \\ Washington, Seattle, WA, USA; \\ ${ }^{2}$ Digestive Disease Center, \\ University of Washington Medical \\ Center, Seattle, WA, USA
}

Correspondence: John E Chandler 2।45 Sheridan Road,

Biomedical Engineering-E310

Evanston, IL 60208, USA

Tel + I 8474673216

Fax + I $84749 \mid 4928$

Email johnchandler20II@u.

northwestern.edu
Purpose: Direct visualization of pancreatic ductal tissue is critical for early diagnosis of pancreatic diseases and for guiding therapeutic interventions. A novel, ultrathin ( $5 \mathrm{Fr}$ ) scanning fiber endoscope (SFE) with tip-bending capability has been developed specifically to achieve high resolution imaging as a pancreatoscope during endoscopic retrograde cholangiopancreatography (ERCP). This device has potential to dramatically improve both diagnostic and therapeutic capabilities during ERCP by providing direct video feedback and tool guidance to clinicians.

Methods: Invasiveness of the new tip-bending SFE was evaluated by a performance comparison to ERCP guide wires, which are routinely inserted into the pancreatic duct during ERCP. An in vitro test model with four force sensors embedded in a synthetic pancreas was designed to detect and compare the insertion forces for $0.89 \mathrm{~mm}$ and $0.53 \mathrm{~mm}$ diameter guide wires as well as the $1.7 \mathrm{~mm}$ diameter SFE. Insertions were performed through the working channel of a therapeutic duodenoscope for the two types of guide wires and using a statistically similar direct insertion method for comparison to the SFE.

Results: Analysis of the forces detected by the sensors showed the smaller diameter $0.53 \mathrm{~mm}$ wire produced significantly less average and maximum forces during insertion than the larger diameter $0.89 \mathrm{~mm}$ wire. With the use of tip-bending and optical visualization, the $1.7 \mathrm{~mm}$ diameter SFE produced significantly less average force during insertion than the $0.89 \mathrm{~mm}$ wire at every sensor, despite its larger size. It was further shown that the use of tip-bending with the SFE significantly reduced the forces at all sensors, compared to insertions when tip-bending was not used.

Conclusion: Combining high quality video imaging with two-axis tip-bending allows a larger diameter guide wire-style device to be inserted into the pancreatic duct during ERCP with improved capacity to perform diagnostics and therapy.

Keywords: endoscopic retrograde cholangiopancreatography (ERCP), scanning fiber endoscope (SFE), pancreatic cancer, minimally invasive therapy

\section{Introduction}

\section{Pancreatic cancer detection}

Pancreatic cancer is currently the fourth most fatal cancer in the United States with a 5-year survival rate of 5\%. In 2009, it was estimated that 35,240 people in the US would die from pancreatic cancer. ${ }^{1}$ Current pancreatic imaging technologies capable of cancer detection and diagnosis include computed tomography (CT), magnetic resonance imaging (MRI), endoscopic ultrasound (EUS), and endoscopic retrograde cholangiopancreatography $(\mathrm{ERCP}) .{ }^{2}$ Contrast enhanced $\mathrm{CT}$ is the primary method used for cancer detection, but magnetic resonance cholangiopancreatography 
(MRCP) and EUS are also commonly used. ${ }^{3}$ With a lack of early stage disease indicators such as biomarkers, these low-resolution imaging methods often fail to detect most cases at a treatable stage of the disease. For high-risk individuals, the lack of biomarkers and other diagnostic aides requires a high-resolution optical imaging device to see the specific changes associated with neoplasia in ductal tissue. For example, main-duct intraductal papillary mucinous neoplasms (IPMNs), which should be endoscopically visible, are a precancerous macroscopic lesion that is considered more aggressive and dangerous than branch-duct IPMNs. ${ }^{4}$ High quality optical imaging within the ductal lumen would also provide direct guidance for interventional tools used for biopsy and therapy. Current clinical practice, however, relies on the use of indirect imaging methods such as $\mathrm{x}$-ray fluoroscopy to assist the doctor. Besides providing much lower resolution structural images, the fluoroscopy exposes both the patient and the operators to unhealthy levels of radiation. ${ }^{5}$ As a result, there is a need to shift from the diagnosis of symptomatic pancreatic cancer to the screening of asymptomatic individuals at high risk using minimally invasive devices. ${ }^{6}$ To accomplish this, technology needs to be developed to implement preemptive and preventative procedures in order to reduce mortality rates for this type of cancer. ${ }^{7}$ Imaging through ERCP and pancreatoscopy is being examined for this purpose, as it is currently the best available means for direct visualization of the pancreatic duct. ${ }^{8,9}$

\section{ERCP guide wires}

Use of guide wires is a common component of ERCP procedures. Wires are used to maintain access to ducts, guide interventional tools for therapy or biopsy, and to aid in cannulation. Many types of wires are available with different diameters, coatings, shapes, and maneuverability. The standard ERCP guide wire is a Teflon-coated stainless steel wire with a flexible coil spring tip. ${ }^{10}$ Other wires have special features which make them ideal for certain situations. These wires can have extra slippery hydrophilic coatings to reduce friction, length markings viewable through the endoscope, kink resistance, different tip stiffness or shape, as well as radiopaque tips for better visualization during fluoroscopy. ${ }^{10}$

\section{Direct visualization of the pancreatobiliary ducts}

The Human Photonics Laboratory (HPL) at the University of Washington has developed a miniature scanning fiber endoscope (SFE), tip shown in Figure 1. ${ }^{11,12}$ This technology makes it possible to construct ultrathin and flexible endoscopes with outer diameters ranging from 3.6 Fr to 5.2 Fr without sacrificing image quality. Full color images are produced by combining red (R-635 nm), green (G-532 nm), and blue (B-442 $\mathrm{nm}$ ) laser light through a cantilevered single mode optical illumination fiber. This fiber is driven into mechanical vibratory resonance at its free end by a tubular piezoelectric actuator, resulting in a spiraling scan pattern. After focusing the scanned RGB-white laser illumination, an image is constructed from backscattered light at the scanned spot. Collection of the backscattered light is achieved by a ring of multimode optical fibers placed around the scanning assembly. With this configuration, the endoscope produces 500 line (200,000 pixels) color images at a $30 \mathrm{~Hz}$ frame rate and $90^{\circ}$ field of view. ${ }^{12}$ Each SFE has a medical grade polyurethane coating and a glass lens unit at the tip for biocompatibility. ${ }^{12}$

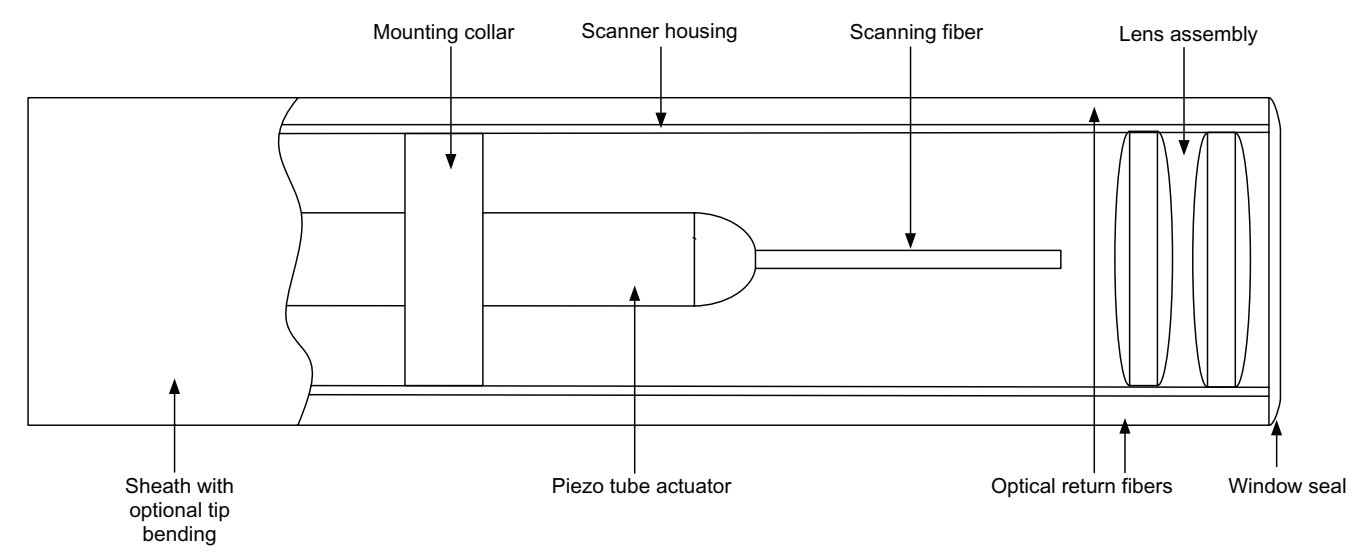

Figure I Schematic of the $9 \mathrm{~mm}$ long distal rigid tip of the SFE. Abbreviation: SFE, scanning fiber endoscope. 
In comparison to the $2.1 \mathrm{~mm}$ diameter pancreatoscope discussed by Kodama et al, the SFE has approximately four times the number of pixels in the acquired images: 200,000 compared to $50,000 .{ }^{8}$ The addition of a working channel further increased the size of this pacreatoscope to $2.4 \mathrm{~mm}$ in diameter, but the $0.5 \mathrm{~mm}$ working channel was not large enough for tissue biopsy. ${ }^{9}$ In addition the SpyGlass ${ }^{\circledR}$ system from Boston Scientific (Natick, MA) as presented by Chen and Pleskow uses a smaller image guide, producing approximately 100-line pixelated images compared to 500-line nonpixelated images produced by the SFE. ${ }^{13}$ With the SpyGlass system, image-guided tissue biopsy is possible with a larger working channel, but the overall size of the scope limits its routine use to the biliary ducts. With smaller high quality imaging devices such as the SFE, future ultrathin pancreatoscope designs may require the imaging scope to be used as a "guide wire with eyes" with hollow biopsy tools designed to run over the guide wire, and saline flushing provided in the annular spacing.

\section{Post-ERCP pancreatitis}

Post ERCP pancreatitis (PEP) is an inflammation of the pancreas that is the primary complication of ERCP, occurring after $4 \%-8 \%$ of all procedures..$^{14} \mathrm{PEP}$ can range from a mild irritation to a severe illness that can lead to organ failure and in some cases death. Examination of the causes of PEP has shown that it is primarily a result of trauma to the pancreatic duct during ERCP. This has been shown to be reduced when devices designed to aid in cannulation are used, such as guide wires and pancreatic stents. ${ }^{14}$ Trauma can also occur due to the injection of contrast media for fluoroscopy. Injection pressure and the amount of fluid injected can both cause damage to the ductal lining. In addition, the composition of contrast media has been found to be chemically irritating to the pancreatic duct and as a result contributes to pancreatitis as well. ${ }^{14}$ Finally, physician skill and experience play significant roles in PEP rates, since ERCP competence requires successful cannulation in more than $90 \%$ of cases and it generally takes between 400 and 500 procedures to reach that skill level. ${ }^{14}$

To further reduce PEP rates as well as improve diagnostic and therapeutic capabilities, it has been predicted that with a device capable of high quality imaging, a single operator system, and two-axis tip deflection, pancreatoscopy will replace pancreatography as the imaging modality of choice during ERCP., ${ }^{2,6,15}$ By using a tip-bending SFE as a "guide wire with eyes," diagnosis can be performed in addition to image-guided interventions without the need for fluoroscopy. However, to preserve high-resolution imaging the new SFE with tip-bending is approximately twice the diameter of the most common ERCP guide wire, so evaluation of its safety is critical. Prior to approaching the pancreas, non-tip-bending versions of the SFE technology have been evaluated as potential "guide wires with eyes" through in vitro human bile duct model studies as well as a successful in vivo insertion into a pig bile duct during ERCP. ${ }^{16,17}$ After success with these previous studies, this experiment evaluated the ability of the SFE to serve as a "guide wire with eyes" for ERCP procedures in the pancreatic duct. In this study, cannulation forces were measured in an in vitro comparison between two sizes of common ERCP guide wires and the prototype steerable SFE. If the larger SFE causes similar insertion forces to ERCP guide wires, then it could provide a safe alternative to the use of fluoroscopy and the injection of radiopaque contrast during many ERCP procedures.

\section{Methods}

Due to the high risk associated with invasive procedures like ERCP, new devices must be carefully evaluated for safety before they can be used. In particular the SFE is being proposed for use in a manner similar to a guide wire, but it has a diameter nearly twice the size of a standard ERCP wire, $1.7 \mathrm{~mm}$ versus $0.89 \mathrm{~mm}$. Despite this, the SFE has the combined capabilities of high quality optical video imaging and two-axis tip deflection, both of which the wire lacks. The relative invasiveness of the SFE and guide wires was determined through the placement of force sensors on an in vitro synthetic model of a pancreas in a human ERCP trainer. A mechanical simulator was chosen for this experiment as they are lower in cost than virtual computer simulators that lack realism and because they do not have the ethical concerns associated with using live animals. ${ }^{18}$ The SFE was determined safe and of equal or lesser invasiveness if its insertion forces were less than or not significantly different from those of the standard ERCP guide wire.

\section{SFE with two-axis tip-bending capability}

A new SFE has been created with the goal of imaging the main pancreatic duct in a minimally invasive manner and providing visualization for therapy. It is capable of producing high quality video imaging equal to that achieved by previous SFE models with the additional capability of two-axis tip deflection. The tip-bending scope used for this study has an outer diameter of $1.7 \mathrm{~mm}$, with a $9 \mathrm{~mm}$ rigid tip and a proximal $10 \mathrm{~mm}$ bend section attached to a $2 \mathrm{~m}$ flexible sheath capable of pushing it into a lumen. Tip-bending is achieved by replacing eight of the optical collection fibers with four 
conduits containing tension wires which increases the overall diameter by $0.1 \mathrm{~mm}$ over a non-steerable SFE. ${ }^{12}$ The direction of tip deflection is controlled using a customized one-handed joystick with a thumb-stick. Potential tip deflection angles are continuous between $0^{\circ}$ and the maximum bend angle and are directly proportional to the amount of deflection applied to the thumb-stick by the operator. Maximum tip deflection angles of $\pm 30^{\circ}$ on both axes were measured when the tether was in tension, completely straight without slack. ${ }^{17}$ When inserted through the working channel of a larger endoscope, average maximum deflection angles were reduced to $\pm 15^{\circ}$ on both axes, as shown in Figure 2. Tip deflection measurements were taken prior to in vitro experiments to determine the maximum angles achievable in the model. Angle measurements were taken using a protractor with $0^{\circ}$ deflection parallel to the SFE tether proximal to the bend section. During operation, there was no feedback to the operator providing a measured tip deflection angle. However, the view observed through the direct video imaging reflects the degree of bending inside a lumen allowing the operator to steer the device appropriately for the observed ductal anatomy.

\section{Synthetic pancreas with force sensors}

A synthetic model of a human pancreatic duct designed to be used with a commercial ERCP trainer (Koken, Tokyo, Japan) was built with integrated force sensors for in vitro comparison of the SFE and ERCP guide wire insertion forces. For an average adult, the length of the pancreas is $15-25 \mathrm{~cm}$, the height is $3-5 \mathrm{~cm}$, and the thickness is $1.5-3.5 \mathrm{~cm} .{ }^{19}$ The pancreas has a basic shape that is designated into sections that include the head, neck, body and tail. The main pancreatic duct is smooth and tapers gradually from head to tail with diameters of 3-4 $\mathrm{mm}$ in the head, $2-3 \mathrm{~mm}$ in the body, and

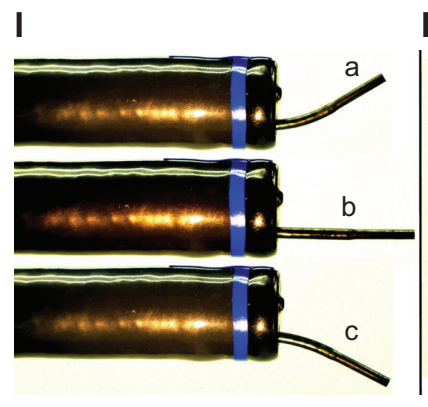

Side view

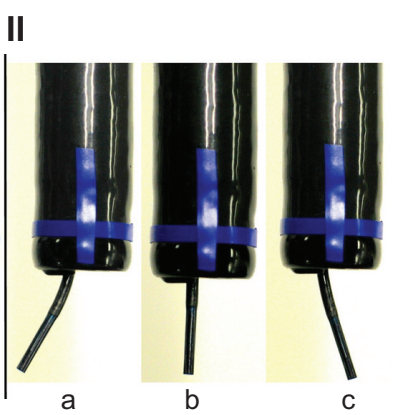

Top view
Figure 2 Demonstration of the two-axis tip-bending capability of the SFE when inserted through the working channel of a larger endoscope. (I) (a-c) side view showing vertical axis deflection of the SFE. (II) (a-c) top view showing horizontal axis deflection of the SFE. Blue tape marks the orientation of the endoscope and all bend angles are approximately $15^{\circ}$.

Abbreviation: SFE, scanning fiber endoscope.
1-2 $\mathrm{mm}$ in the tail. ${ }^{19}$ It was assumed for this experiment that the pancreas was $15 \mathrm{~cm}$ long and that the diameter of the pancreatic duct tapered from $3 \mathrm{~mm}$ in the head to $2 \mathrm{~mm}$ in the body to $1 \mathrm{~mm}$ at the tip of the tail.

To build the in vitro synthetic pancreas model, an expendable mold of the pancreatic duct shape was created out of polymer modeling clay (Sculpey, Polyform Products Co, Elk Grove Village, IL) and baked to harden it. PlatSil Gel-10 (Polytek Development Corp, Easton, PA), a room temperature vulcanizing (RTV) silicone rubber intended for use as synthetic tissue, was layered onto the mold three times to produce a duct thickness of approximately $1 \mathrm{~mm}$. Piezoelectric fluoropolymer film sensors [LDT1-028 K] (Measurement Specialties Inc, Hampton, VA) were chosen to measure the stresses induced on the pancreatic duct during ERCP guide wire insertion. Due to their very high strain sensitivity of about $12 \mathrm{mV} / \mu$ strain $(14.4 \mathrm{~V} / \mathrm{N})$, these sensors can detect very small mechanical deformations resulting from the contact force between the guide wire tip and the duct. Four of these piezoelectric force sensors were wrapped around the synthetic duct at the observed locations that provided distinct sensor readings from the most commonly impacted locations as shown in Figure 3. These locations were determined via experimentation with the sensor positions and insertion of guide wires into several of the early duct prototypes. Once the sensors were in place, more PlatSil Gel-10 was applied to create a synthetic pancreas that would support and protect the duct with approximate outer dimensions of $15 \mathrm{~cm} \times 4 \mathrm{~cm} \times 2 \mathrm{~cm}$. In order to mount the pancreas securely in the ERCP trainer, the mold was encased in a plexiglass box, with the duct opening extending from one end to connect with the ERCP trainer.

\section{Signal conditioning circuit and computer interface}

An analog signal conditioning circuit was constructed to measure the voltage produced by the piezoelectric film sensors. The circuit design was based on a circuit designed for the Piezo Film Pulse Sensor project. ${ }^{20}$ Charge leakage between the two sides of the piezoelectric sensors makes it difficult to measure quasi-static strains. Thus, the circuit used very high impedance for the sensors enabling low-frequency measurement. In addition, the circuit contains a three-pole low-pass filter with a cutoff frequency of around $12 \mathrm{~Hz}{ }^{20}$ A low-pass filter was used to eliminate the high frequency noise, including $60 \mathrm{~Hz}$ line interference. The sensors were shielded from capacitance noise using a grounded foil shield and coaxial cable for the signal wires. To record the forces 


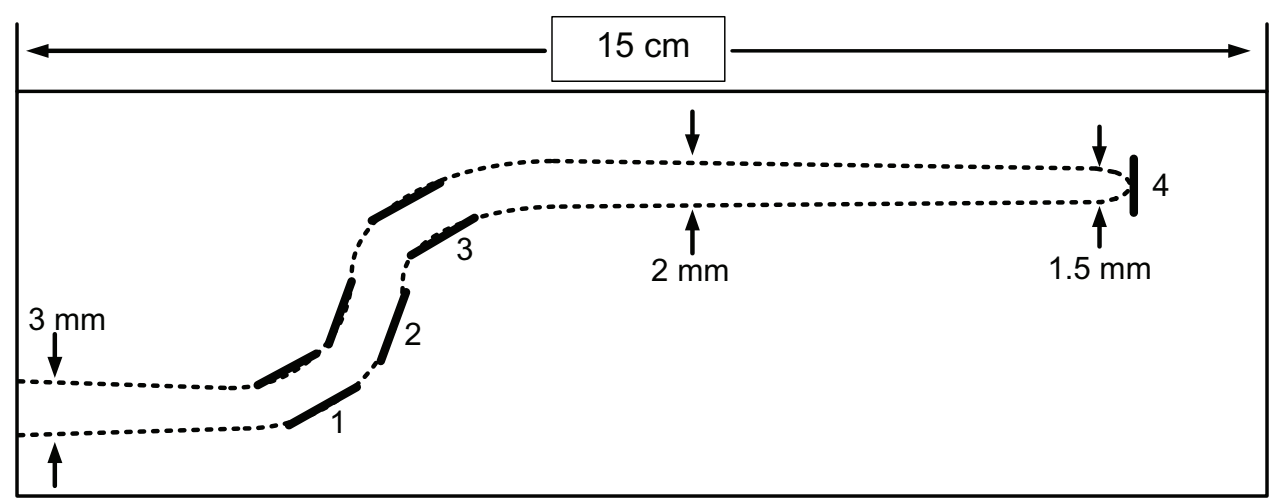

Figure 3 Cross-sectional view of the synthetic pancreas along the main pancreatic duct with force sensor locations, no I-4 marked head to tail. The duct is distensible allowing the SFE to be inserted far enough to trigger Sensor 4.

Abbreviation: SFE, scanning fiber endoscope.

during insertion, each sensor was connected to a dedicated circuit board and interfaced with Labview (National Instruments, Austin, TX) via a National Instruments 6009 USB card. For each sensor, real-time root mean square (RMS) output voltages were recorded to a data file.

\section{Test setup}

All testing of the guide wires and tip-bending SFE utilized the synthetic ERCP trainer model. The synthetic pancreas with embedded sensors was attached to the ERCP trainer so that guide wires and the SFE could be inserted into the pancreatic duct using an ED-3470TK therapeutic ERCP duodenoscope (Pentax Corp, Tokyo, Japan) connected to an EPK 1000 video processor (Pentax Corp) as shown in Figure 4.

This therapeutic ERCP duodenoscope had a $4.2 \mathrm{~mm}$ diameter working channel and an elevator mechanism to direct the devices sideways toward duct openings. Initial force evaluation experiments for the insertions of standard ERCP guide wires into the pancreatic duct were conducted by an expert endoscopist. Each wire was inserted through the working channel of the ERCP duodenoscope all the way to the end of the synthetic pancreatic duct, and forces were recorded during the insertion and removal. A standard $0.89 \mathrm{~mm}$ diameter ERCP guide wire (Cook Medical, Bloomington, IN) was tested as well as a $0.53 \mathrm{~mm}$ diameter Tracer MetroDirect guide wire (Cook Medical). Insertion of the two guide wires was assisted through the use of ERCP cannulas (Bard, Murray Hill, NJ). During these initial experiments, engineers from the Human Photonics Lab were also trained by the expert endoscopist to perform further experimental insertions. All the guide wire insertions were performed blind without the aid of any direct or indirect visualization beyond the camera on the duodenoscope. The data used for statistical analysis and comparison of the devices was collected after sufficient training and practice with each device, such that statistically similar results were obtained on insertions with the same device.

\section{Direct insertion method}

Insertion of the tip-bending SFE was attempted by engineers in the same manner as the guide wires, through the working channel of the duodenoscope. Attempts to fully insert the SFE through the duodenoscope were unsuccessful due to friction in the synthetic model and the difficulty of pushing the SFE by its flexible tether from outside the duodenoscope. This friction arose because the synthetic interior of the duct did not mimic the friction produced by a liquid in vivo environment. During previous in vivo studies where the SFE was inserted through a duodenoscope, friction has not been a problem. ${ }^{16}$

To insert the SFE into the in vitro model, a direct insertion method had to be developed that did not require the use of the duodenoscope, as shown in Figure 5. To simulate entrance into the duct through the elevator of a duodenoscope, a $16 \mathrm{~cm}$ length of $2 \mathrm{~mm}$ inner diameter nylon tubing with a $90^{\circ}$ bend was attached to the synthetic pancreas. This setup was designed to produce statistically similar results to those achieved during insertions with the duodenoscope, specifically at the first sensor, where significantly higher forces were observed when directly inserting without the tubing. Using this direct insertion method, insertions of the SFE were performed with and without tip-bending to evaluate the impact of steering on the forces produced by the SFE. The direct method of insertion was also used to compare the SFE to both guide wires. Optical visualization of the duct was used during all SFE insertions and the SFE could be fully inserted in this manner, but insertion was stopped once the tail of the duct could be clearly visualized. 


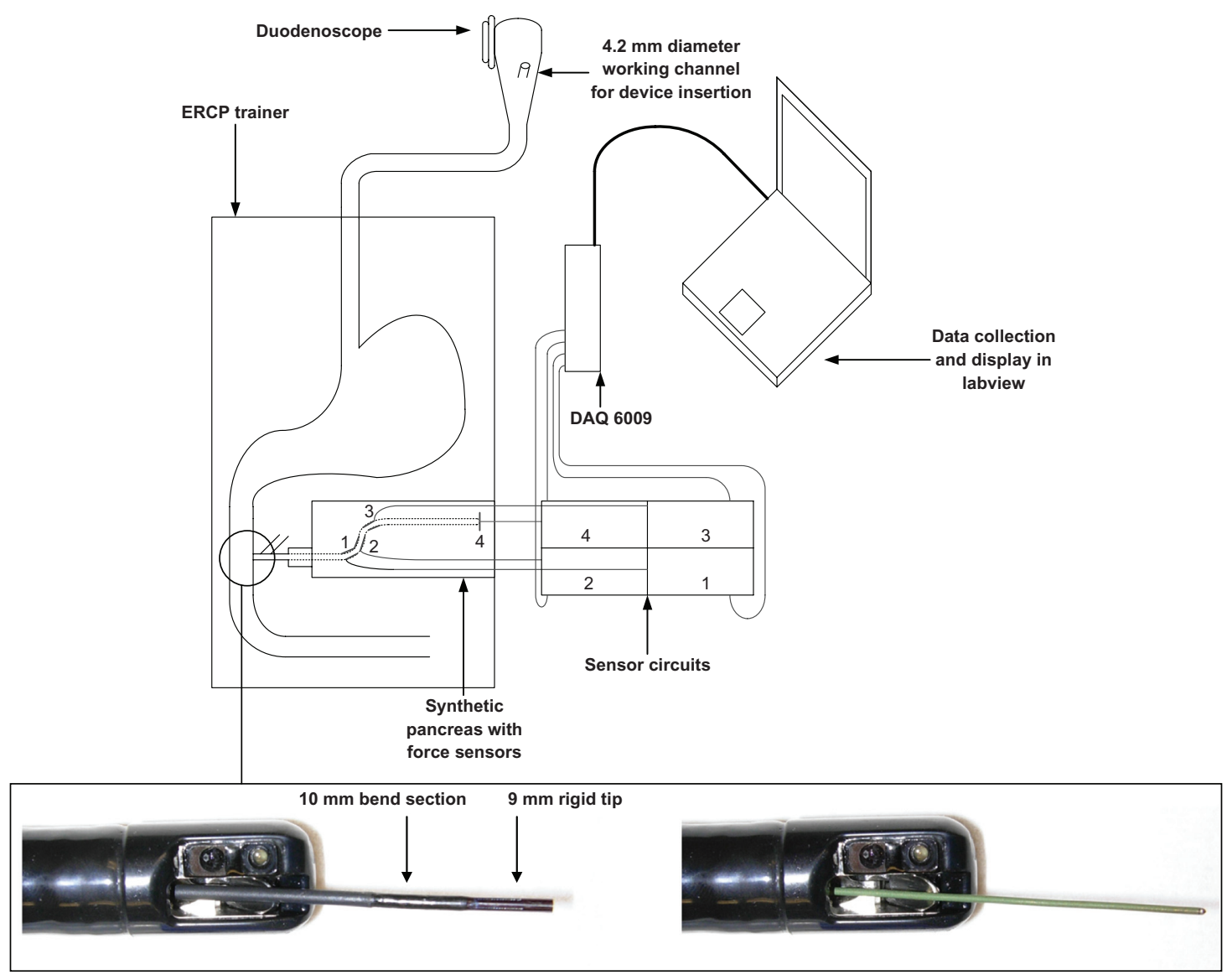

Figure 4 Experimental setup (top) including the synthetic pancreas with force sensors and DAQ card to record the output voltages. A duodenoscope is used to introduce the SFE (bottom left) and $0.89 \mathrm{~mm}$ diameter guide wire (bottom right) into the model.

Abbreviations: SFE, scanning fiber endoscope; DAQ, data acquisition.

Navigation of the SFE within the model was done by two engineers, one to steer the device and one to push the device forward into the lumen. Calibration of the bending directions could be done in the SFE user interface after initial insertion by rotating the image to match the bending directions on the thumb-stick. Tip-bending was used to minimize contact of the distal tip of the SFE with the duct walls and to assist in navigating corners. To navigate a bend, the operators attempted to maintain the position of the tip of the SFE centrally within the lumen using the video imaging to guide the amount of bending required to achieve this while the device was advanced. Sharper bends within the duct correspondingly required larger tip-bending angles. Amount of bending and bending location were determined on a case-by-case basis by the operators to simulate multiple clinical procedures in patients with unknown anatomy. Approximately $15^{\circ}$ of bending were available on both axes during insertions with the SFE. All tip-bending results were recorded under conditions of proper operation, minimizing direct contact with lumen walls. Improper operation would increase the forces caused by the SFE. On the SFE insertions that did not use tip-bending, the device was treated as a guide wire with the tip oriented at $0^{\circ}$.

\section{Data collection, analysis, and device comparison}

With the $0.89 \mathrm{~mm}$ guide wires, data was collected on a total of 44 successful insertions performed with the duodenoscope and on 46 total insertions via the direct insertion method. For the $0.53 \mathrm{~mm}$ wire, data was collected on seven successful insertions with the duodenosocpe and 34 using the direct insertion method. With the SFE a total of eight tip-bending insertions and 18 insertions without bending were performed, all through the direct insertion method. More insertions were performed with the $0.89 \mathrm{~mm}$ wire than the other two devices as this device was used as a standard for determining similarity between the two insertion methods. Other differences in the number of trials with each device were due to device availability.

RMS voltage signals were analyzed using MATLAB (MathWorks, Natick, MA). Force measurements below the 


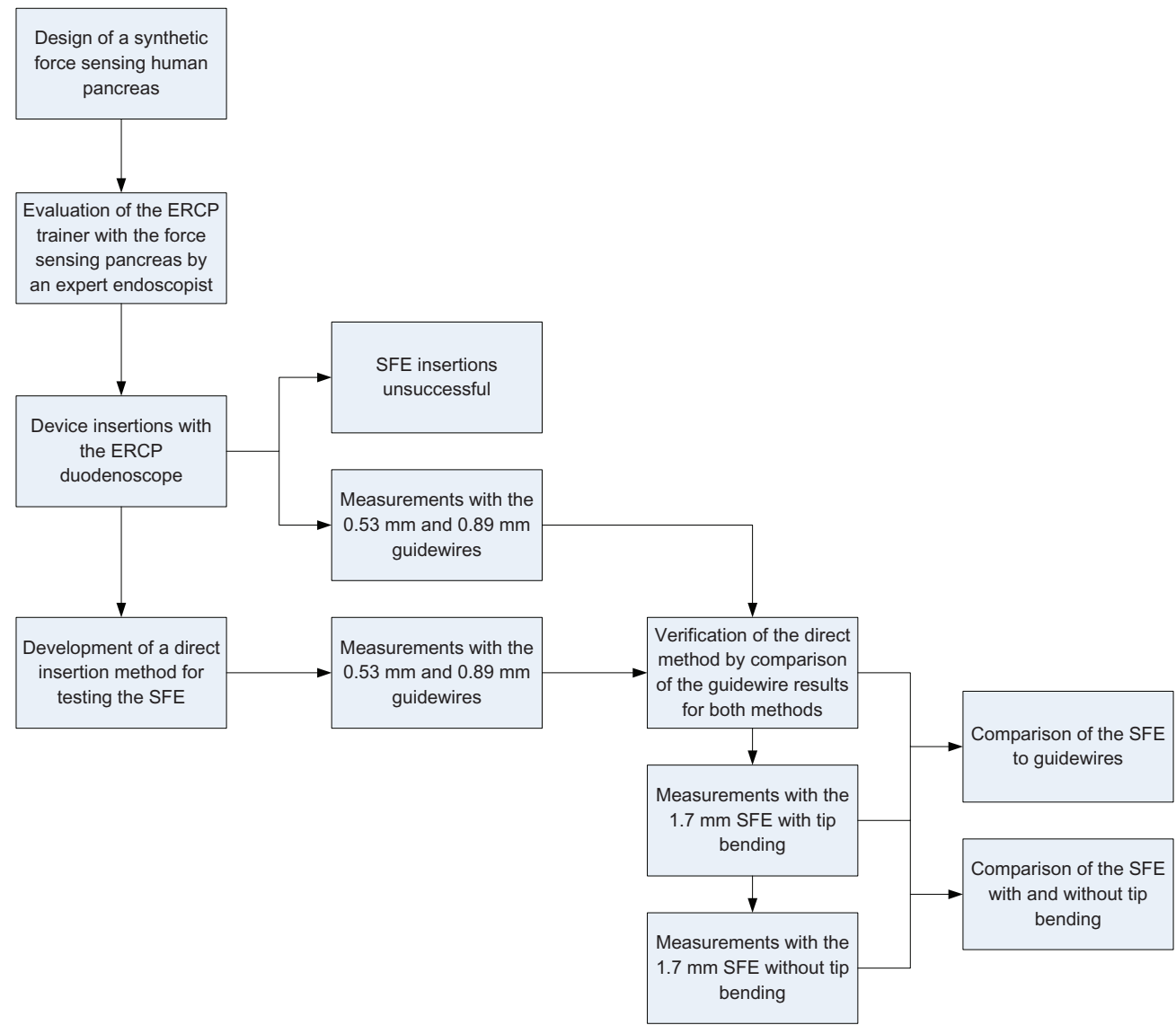

Figure 5 Stages of development for the experimental testing methods.

Abbreviations: SFE, scanning fiber endoscope; ERCP, endoscopic retrograde cholangiopancreatography.

noise level of the sensors $(0.02 \mathrm{~V})$ were not considered. For each sensor, the non-zero average force response and maximum force response were computed. Statistical comparison of both the average and maximum forces was performed with a paired $t$-test at a $95 \%$ significance level using the MATLAB statistics toolbox. Verification of the calculated results was performed by visual inspection of the plotted RMS response curves for each device.

Measurements of the maximum normal contact forces for the two guide wires and the SFE were taken with a Shimpo Digital Force Gauge (Shimpo Instruments, Kyoto, Japan). These values were used to determine the maximum force threshold which each device could apply to the duct. Each device was applied normally to the contact plate in the same manner used during experimental insertion and the maximum force applied was recorded before buckling or deflection. Average maximum force thresholds recorded had the $0.89 \mathrm{~mm}$ guide wire at $0.201 \mathrm{~N}$, the $0.53 \mathrm{~mm}$ guide wire at $0.120 \mathrm{~N}$, and the SFE at $0.952 \mathrm{~N}$. These values corresponded to average maximum normal stresses of $323.175 \mathrm{kPa}$ for the $0.89 \mathrm{~mm}$ guide wire, $536.119 \mathrm{kPa}$ for the $0.53 \mathrm{~mm}$ guide wire, and $419.596 \mathrm{kPa}$ for the SFE. The stress level thresholds place the SFE between the two clinically accepted devices.

\section{Results Verification of the direct insertion method} Similarity of the direct insertion method to the duodenoscope insertion method was verified by comparing both methods using the $0.89 \mathrm{~mm}$ guide wire and the $0.53 \mathrm{~mm}$ guide wire. As shown in Table 1, there was no statistical difference between the average forces for the two methods with the $0.89 \mathrm{~mm}$ guide wire at sensors 1,3 , and 4 , but the direct method had higher average force at sensor 2 . For the maximum force measurements with the $0.89 \mathrm{~mm}$ wire there was no difference between the methods at sensors 1 and 4 , while the direct method had higher maximum force at sensors 2 and 3 .

With the $0.53 \mathrm{~mm}$ wire, Table 1 shows there was no difference in average force for the two methods at sensor 1 while the direct method was higher at 2 and 3, but less at sensor 4. For the $0.53 \mathrm{~mm}$ wire no difference in maximum force was observed at sensor 1, while the direct method was higher at 2 and 3, but less at sensor 4 . 
Table I T-statistic comparisons of the duodenoscope and direct insertion methods

\begin{tabular}{|c|c|c|c|c|c|c|}
\hline t-test & Measurement & $\begin{array}{l}\text { Confidence } \\
\text { interval }\end{array}$ & $\begin{array}{l}\text { Sensor I } \\
\text { (head) }\end{array}$ & Sensor 2 & Sensor 3 & $\begin{array}{l}\text { Sensor } 4 \\
\text { (tail) }\end{array}$ \\
\hline $0.89 \mathrm{~mm}$ diameter guidwire (direct) & Average force & \pm 2.00 & -0.1998 & 5.4234 & 1.5836 & 1.5962 \\
\hline $\begin{array}{l}\text { versus } 0.89 \mathrm{~mm} \text { diameter guide wire } \\
\text { (duodenoscope) }\end{array}$ & Maximum force & \pm 2.00 & 0.0267 & 4.48643 & 4.7384 & 0.5906 \\
\hline $0.53 \mathrm{~mm}$ wire (direct) versus $0.53 \mathrm{~mm}$ & Average force & \pm 2.06 & 0.9705 & 2.6174 & 4.2837 & -2.7657 \\
\hline wire (duodenoscope) & Maximum force & \pm 2.06 & 2.0209 & 3.2278 & 3.7382 & -2.339 \\
\hline $0.53 \mathrm{~mm}$ wire (duodenoscope) versus & Average force & \pm 2.01 & -3.4959 & -2.6933 & $-1.203 \mid$ & -1.2807 \\
\hline $0.89 \mathrm{~mm}$ wire (duodenoscope) & Maximum force & \pm 2.01 & -5.6858 & -3.5772 & $-2.6|4|$ & -1.7835 \\
\hline
\end{tabular}

In addition, the $0.53 \mathrm{~mm}$ and $0.89 \mathrm{~mm}$ guide wires were compared to each other using both insertion methods. Table 1 and Figure $6 \mathrm{~A}$ show that both methods determined that the smaller, $0.53 \mathrm{~mm}$ diameter, wire produced less average and maximum force than the larger, $0.89 \mathrm{~mm}$ diameter, wire. The direct method was verified to test the SFE for this experiment because there was not a significant difference between the forces detected by the two methods specifically at the point of insertion (Sensor 1). Sensor 1 was used as the verification location because it was affected by the insertion method more than any other sensor. Multiple insertion methods were

A $0.53 \mathrm{~mm}$ guidewire vs $0.89 \mathrm{~mm}$ guidewire

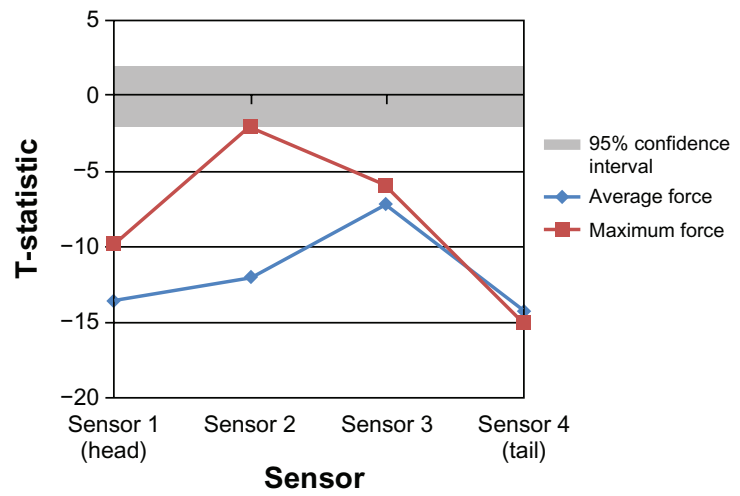

C SFE vs $0.53 \mathrm{~mm}$ guidewire

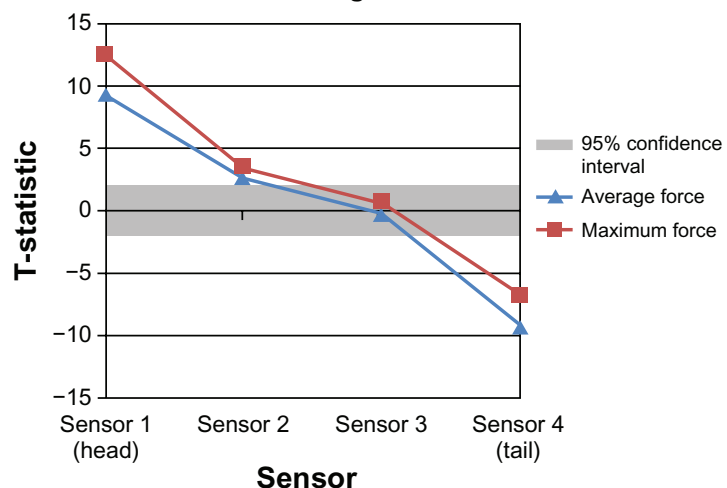

attempted before selecting the direct method described and each had significantly different forces at sensor 1 , while results at sensors 3-4 were generally consistent. Also, both methods produced the same result when comparing the two different diameter guide wires.

\section{Device comparisons with the direct insertion method}

Plots showing the results of the $t$-tests for both average and maximum forces with the direct insertion method are shown in Figure 6. The 95\% confidence interval is shown on each

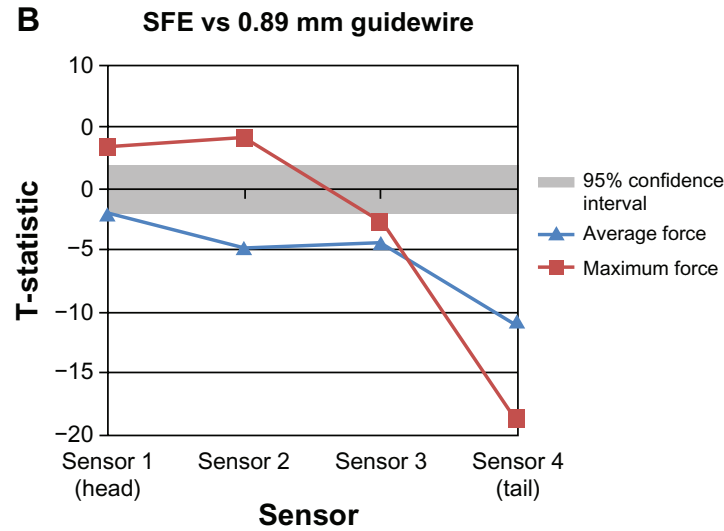

\section{SFE vs SFE without tip bending}

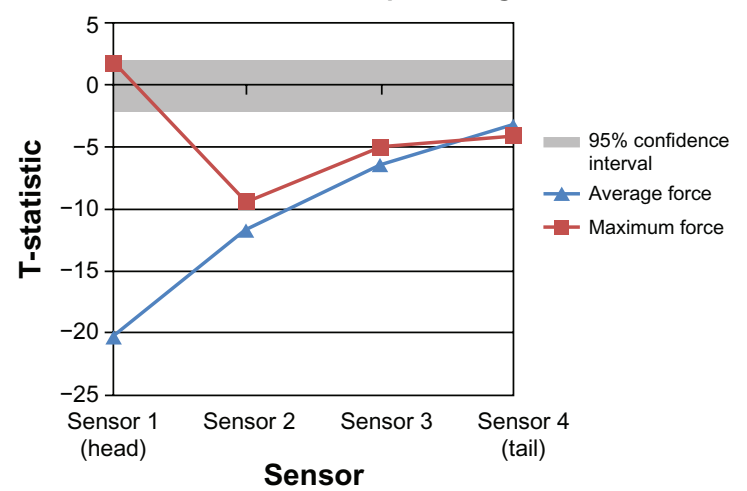

Figure 6 Average force and maximum force $t$-test result plots with the direct insertion method: (A) 0.53 mm diameter guide wire versus 0.89 mm diameter guide wire, (B) SFE versus $0.89 \mathrm{~mm}$ diameter guide wire, (C) SFE versus $0.53 \mathrm{~mm}$ diameter guide wire (D) SFE versus SFE without tip-bending.

Abbreviation: SFE, scanning fiber endoscope. 
plot along with the values of the $t$-statistic computed at each sensor. The device being tested is always listed first, with the comparison device listed second. $T$-statistic values above the confidence interval indicate significantly higher maximum or average forces for the tested device. Values below the confidence interval indicate that the tested device had significantly lower average or maximum forces than the comparison device. $T$-statistic values inside the confidence interval indicate that there was not a significant difference between the forces of the two devices.

From Figure 6A it can be seen that the $0.53 \mathrm{~mm}$ wire had significantly less average and maximum force at all sensors compared to the $0.89 \mathrm{~mm}$ wire. Insertion times were comparable for both devices. Figure $6 \mathrm{~B}$ shows that the SFE had similar average force at sensor 1 and less aveage force at sensors 2, 3, and 4 than the $0.89 \mathrm{~mm}$ wire. The SFE also had higher maximum force at sensors 1 and 2, but less at sensors 3 and 4. On both the average force plot and the maximum force plot, the SFE appears to become relatively less invasive compared to the guide wire as it moves from head to tail of the duct. SFE insertions took approximately 3 times longer than those for the $0.89 \mathrm{~mm}$ guide wire. In comparison to the $0.53 \mathrm{~mm}$ guide wire, as shown in Figure $6 \mathrm{C}$, the SFE had higher average and maximum forces at sensors 1 and 2 , similar forces at 3 , and lower forces at sensor 4 . With increasing distance from the head of the duct, the relative invasiveness of the SFE compared to the wire decreased for both average and maximum forces. SFE insertions took approximately 3.5 times longer than those for the $0.53 \mathrm{~mm}$ guide wire. The observed reasons for the large differences in procedure time for the SFE compared to both diameter wires were twofold. First the entire length of the tether of the SFE was highly flexible making it prone to deflection when pushed into a lumen. Second, both guide wires had coatings which reduced friction in the model, whereas the polyurethane sheathing of the SFE generated significant friction in the synthetic model. These two combined factors made the SFE difficult to insert and push into the synthetic pancreas. Comparing the SFE to the SFE without tip-bending, Figure 6D shows that the SFE had significantly less average force at all sensors when tip-bending was used. Also, significantly less maximum force was recorded with tip-bending at sensors 2,3 , and 4 . The effects of tip-bending on the difference in average force decreased as the device moved from the head to the tail of the duct.

\section{Discussion}

\section{Tip-bending for small scale video endoscopes and guide wires}

Use of the direct method allowed for direct comparison of the SFE when tip-bending was and was not used.
This comparison allowed for the effects of tip-bending to be determined with all other variables held constant. During these insertions it was observed by the operators that the SFE felt easier to insert with the use of tip-bending. Also, the operators observed fewer impacts with the lumen walls when tip-bending was used compared to when it was not. Figure $6 \mathrm{D}$ supported these observations showing that tip-bending resulted in significantly less average force at all sensors and significantly less maximum force at all sensors except the first where there was no difference. These results are similar to the observations from Kodama et al that tipbending on a small pancreatoscope not only increases the observable field, but also allows insertion of the device all the way to the tail of the duct. ${ }^{8}$ Figure $6 \mathrm{D}$ also indicates that the use of tip-bending created the greatest difference in average force at the first sensor and the difference decreased further into the duct. This result was unexpected as this shows tip-bending being less effective at reducing force in narrower portions of the duct. However, it was observed through the video imaging during use of the SFE that narrower ducts that were only slightly larger in diameter than the SFE tended to naturally guide the device obviating the need for steering. By comparison, in wider portions of the duct tip-bending was very effective at minimizing contact and reducing impacts and friction along the duct walls. Maximum measured forces during the tip-bending insertions occurred most frequently during removal of the device from the duct. This observation further supports the impact of tip-bending on reducing forces as the greatest forces occurred when tipbending could not be used effectively during withdrawal of the device from the duct.

\section{Comparing the SFE to current guide wire technology}

The comparison of the two common ERCP guide wires showed that the smaller $0.53 \mathrm{~mm}$ diameter guide wire was less invasive in terms of both average and maximum force during insertion as compared to the larger $0.89 \mathrm{~mm}$ diameter guide wire. This was demonstrated with both insertion methods as shown by Table 1 and Figure 6A. Both of these devices were inserted blind without the aid of direct or indirect visualization and had comparable insertion times. Comparison of the SFE invasiveness to that of guide wires is difficult as it took longer to insert the SFE due to friction inside the model. This was addressed directly in the analysis by examining maximum force values in addition to averages to remove any potential skewing due to increased procedure time. Also, all points of zero force were removed during the 
data analysis process eliminating points that did not contribute to the results during lengthy procedures.

The analysis obtained under these conditions indicates the SFE with tip-bending produced comparable forces to a device approximately half its size. Figure $6 \mathrm{~B}$ shows the average force created by the SFE during insertion was similar to or less than that of the $0.89 \mathrm{~mm}$ guide wire at all sensors although it had higher maximum forces at the first two sensors. Also, Figure 6B shows that the SFE actually had significantly less average force at the last two sensors and both average and maximum force tended to decrease in the smaller regions of the duct. This indicates the relative invasiveness of the larger device with steering and imaging decreased compared to that of a smaller guide wire as the diameter of the duct decreased. In addition to the $0.89 \mathrm{~mm}$ diameter wire, the SFE was compared to the $0.53 \mathrm{~mm}$ wire. Figure 6C shows that the SFE was more invasive in terms of both average and maximum force at the first two sensors. However, the SFE had comparable forces at the third sensor and less at the last. Again the trend was observed that the relative invasiveness of the SFE compared to the guide wire tended to decrease as the duct got smaller in diameter. In narrower portions of the duct, both guide wires were still much smaller than the duct diameter and therefore would still deflect and buckle off of the walls much more than a larger device, particularly one capable of tip-bending. As a result guide wires tended to have more direct contact with the duct walls and relied on deflection off of the walls for steering. The SFE, in comparison, could stay centered in the duct, minimizing impact with the walls, and preventing deflection. Also, because the SFE could image ahead of its distal end, the end of the duct could be visualized and insertion halted before the SFE had reached it. As a result, the SFE very rarely triggered the final sensor and tended to produce less force as the duct got narrower compared to the guide wires.

\section{Potential use of the SFE as a "guide wire with eyes"}

This experiment demonstrated the potential of the SFE technology to serve as a minimally invasive diagnostic and therapeutic tool during ERCP procedures. Video guided tip-bending with two-axis thumb stick control was shown to significantly reduce the insertion forces of larger diameter guide wire style devices. Steering these devices with tip-bending is necessary to reduce trauma and prevent the complications associated with large diameter devices, such as pancreatitis. Considering this result, larger diameter "guide wires with eyes" such as the SFE can potentially be used to perform pancreatoscopy during ERCP in a comparable manner to a standard blind guide wire used during the procedure. Such procedures would be capable of providing high quality optical imaging of pancreatic ductal tissue for both diagnosis and therapy. Figure 7 shows a video frame captured during insertion of the SFE into the synthetic pancreatic duct demonstrating this capability. In this image, the features of the synthetic duct can be clearly observed including the presence of one of the force sensors wrapped around the duct. With these capabilities the SFE technology has the potential to improve the diagnostic capabilities of ERCP, especially for main duct IPMNs where cell sampling for cytology helps predict malignancy. ${ }^{21}$

\section{Possible improvements for future force analysis in small ducts}

For future in vitro force analysis studies in small ducts, further improvements could be made with the force sensing equipment chosen for the experiment. Specifically, conformable sensor arrays, composed of many individual sensing elements have ideal characteristics for this application. These types of sensor arrays can be made to conform to the overall shape of the duct allowing scanning of the entire ductal area, significantly increasing the number of force sensing locations. ${ }^{22}$ With a sensing array around the entire duct, force locations as well as magnitudes are known, allowing for differentiation between shear and radial forces.

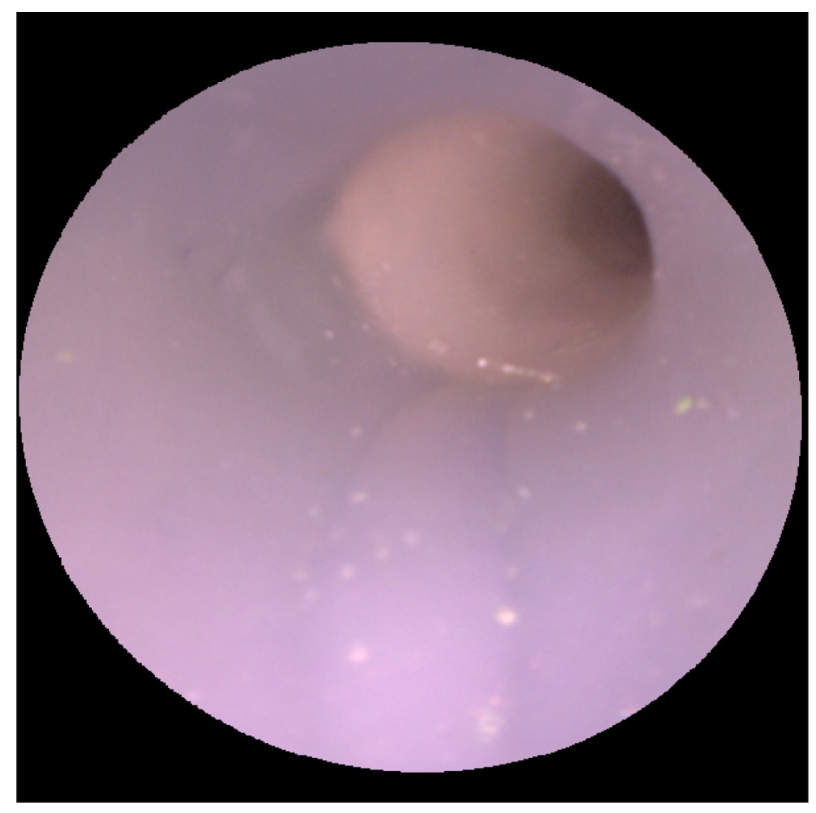

Figure 7 Video frame taken with SFE inside the synthetic pancreatic duct with sensor visible as region of lighter contrast.

Abbreviation: SFE, scanning fiber endoscope. 
Array sensors can also generate force profiles throughout the duct for device insertions and have the capability to perform three dimensional scanning resulting in pressure profiles of the whole duct area. Data from these sensors would improve the quality of comparisons between dissimilar devices, reducing the effects of insertion time and device design differences on the results by providing a complete force profile for each device throughout the entire duct. ${ }^{22}$ The disadvantages of this sensing technology include increased cost and complexity for both the experimental apparatus and data analysis.

In clinical practice, the incidence of post-ERCP pancreatitis increases incrementally with increasing number of attempts at entering the duct as well as with multiple injections of contrast fluid, resulting in longer procedure times. ${ }^{14,23}$ In comparison, high insertion times with the SFE in the in vitro model were due to difficulty pushing the SFE forward in the ductal lumen. This can be attributed to the synthetic materials used for the ERCP model not accurately replicating the friction encountered by devices in a liquid in vivo environment. Achieving a more realistic in vitro model would be expected to reduce the overall friction allowing insertion of the SFE by the duodenoscope method. With such a model, the SFE would be expected to have similar insertion times to guide wires providing a more informative procedure-time comparison to clinical practice.

\section{Possible improvements to the tip-bending SFE for use in small ducts}

Improvements should be made to the SFE that would provide improved results in comparison to current devices and practices. The current design of the tip-bending SFE can be reduced in overall diameter to $1.5 \mathrm{~mm}(-10 \%)$. While still a large diameter device for pancreatoscopy, cannulation aids such as stents can be utilized to prevent complications with smaller duct diameters. Although smaller imaging bundles are possible using coherent fiber-optic technology, the image quality is severely compromised. ${ }^{12}$ In addition, the pull-wire designs for steerable catheters used in vascular surgery are significantly larger in diameter and would require more x-ray fluoroscopic imaging to be useful endoscopically compared to a tip bending pancreatoscope such as the SFE. ${ }^{24}$ Designing the SFE with a less flexible shaft would further reduce insertion time and increase the feasibility of using a duodenoscope to insert the device. With this change, the flexible tip could be left unchanged, but behind this tip, use of a stiffer more robust shaft material would provide increased control and push-ability to the operator without increasing size or stiffness in the distal end within the pancreatobiliary ducts. Performance of the bending mechanism can be improved to consistently achieve or increase the $30^{\circ}$ maximum tip deflection achieved with this device.

\section{Conclusions}

The SFE proved to be a promising medical technology for pancreatoscopy procedures during simulated ERCP. Combining optical visualization with tip-bending allowed the SFE to be inserted into a synthetic pancreatic duct with forces comparable to a guide wire half its size. Compared to the standard $0.89 \mathrm{~mm}$ guide wire the SFE was able to be inserted into the synthetic in vitro ERCP model with less average force per insertion. It was also shown that tip-bending guided by video imaging results in significantly reduced insertion forces caused by inserting small endoscopes into the pancreatic duct. Further evaluation of the SFE's effectiveness for human subjects can now be pursued through in vivo studies. Optimization of the SFE for these studies should permit efficient, minimally invasive diagnostic and therapeutic pancreatoscopy procedures without reliance on fluoroscopy.

\section{Acknowledgments}

Funding for this work was provided by NSF (CBET-0809070, PI-Seibel), NIH (NIBIB 1RC1 EB010900, PI-Seibel), and the Crane Research Experience for Undergraduates at the University of Washington College of Engineering (Chandler). Project advising and guidance provided by Professor Per Reinhall and Rich Johnston. Manuscript editing was provided by Dr Tim Soper. Initial design and fabrication of the in vitro model was done by Ms Megan Leone with advice from Dr Michael Kimmey.

\section{Disclosure}

The authors report no conflicts of interest in this work.

\section{References}

1. Jemal A, Siegel R, Ward E, Hao Y, Xu J, Thun MJ. Cancer statistics, 2009. CA Cancer J Clin. 2009;59(4):225-249.

2. Nichols MT, Russ PD, Chen YK. Pancreatic imaging: current and emerging technologies. Pancreas. 2006;33(3):211-220.

3. NIH state-of-the-science statement on endoscopic retrograde cholangiopancreatography (ERCP) for diagnosis and therapy. NIH Consens State Sci Statements. 2002;19(1):1-26.

4. Terris B, Ponsot P, Paye F, et al. Intraductal papillary mucinous tumors of the pancreas confined to secondary ducts show less aggressive pathologic features as compared with those involving the main pancreatic duct. $A m$ J Surg Pathol. 2000;24(10):1372-1377.

5. Naidu LS, Singhal S, Preece DE, Vohrah A, Loft DE. Radiation exposure to personnel performing endoscopic retrograde cholangiopancreatography. Postgrad Med J. 2005;81(960):660-662.

6. Canto MI. Screening and surveillance approaches in familial pancreatic cancer. Gastrointest Endosc Clin N Am. 2008;18(3):535-553.

7. Kellof GJ, Sullivan DC, Baker H, et al. Workshop on imaging science development for cancer prevention and preemption. Cancer Biomark. 2007;3(1):1-33. 
8. Kodama T, Koshitani T, Sato H, et al. Electronic pancreatoscopy for the diagnosis of pancreatic diseases. Am J Gastroenterol. 2002:97(3): $617-622$.

9. Kodama T, Tatsumi Y, Sato H, et al. Initial experience with a new peroral electronic pancreatoscope with an accessory channel. Gastrointest Endosc. 2004;59(7):895-900.

10. Jacob L, Geenen JE. ERCP guide wires. Gastrointest Endosc. 1996; 43(1):57-60.

11. Seibel EJ, Brown CM, Dominitz JA, Kimmey MB. Scanning single fiber endoscopy: a new platform technology for integrated laser imaging, diagnosis, and future therapies. Gastrointest Endosc Clin N Am. 2008;18(3):467-478.

12. Lee CM Engelbrecht CJ, Soper TD, Helmchen F, Seibel EJ. Scanning fiber endoscopy with highly flexible, $1 \mathrm{~mm}$ catheterscopes for widefield, full-color imaging. J Biophotonics. 2010;3(5-6):385-407.

13. Chen YK, Pleskow DK. SpyGlass single-operator peroral cholangiopancreatoscopy system for the diagnosis and therapy of bile-duct disorders: a clinical feasibility study(with video). Gastrointest Endosc. 2007;65(6):832-841.

14. Abdel Aziz AM, Lehman GA. Pancreatitis after endoscopic retrograde cholangiopancreatography. World J Gastroenterol. 2007;13(19): 2655-2668.

15. Seibel EJ. $1 \mathrm{~mm}$ catheterscope. Proc SPIE. 2008;6852; 685207-685208.

16. Seibel, EJ, Brentnall TA, Dominitz JA. New endoscopic and cytologic tools for cancer surveillance in the digestive tract. Gastrointest Endosc Clin N Am. 2009;19(2):299-307.
17. Lee CM, Chandler JE, Seibel EJ. Wide field fluorescence imaging in narrow passageways using scanning fiber endoscope technology. Proc SPIE. 2010;7558:755806-1-10.

18. Heller SJ, Tokar JL. Current status of advanced gastrointestinal endoscopy training fellowships in the United States. Adv Med Educ Pract. 2011;2:25-34.

19. Hadidi A. Pancreatic duct diameter: sonographic measurement in normal subjects. J Clin Ultrasound. 1983;11(1):17-22.

20. Peterson WD, Skramsted DA, Glumac DE. Phoenix Ambulatory Blood Pressure Monitor Project: Piezo Film Pulse Sensor [homepage on the Internet]. c2005. Available from: http://www.phoenix.tc-ieee. org/004_Piezo_Film_Blood_Flow_Sensor/Phoenix_PiezoPulse.htm. Accessed November 24, 2011.

21. Genevay M, Mino-Kenudson M, Yaeger K, et al. Cytology adds value to imaging studies for risk assessment of malignancy in pancreatic mucinous cysts. Ann Surg. October 28, 2011. [Epub ahead of print.]

22. Dogramadzi S, Virk GS, Bell GD, Rowland RS, Hancock J. Recording forces exerted on the bowel wall during colonoscopy: in vitro evaluation. Int J Med Robot. 2005;1(4):89-97.

23. Bailey A, Bourke M, Williams S, et al. A prospective randomized trial of cannulation technique in ERCP: effects on technical success and post-ERCP pancreatitis. Endoscopy. 2008;40(4):296-301.

24. Fu Y, Liu H, Huang W, Wang S, Liang Z. Steerable catheters in minimally invasive vascular surgery. Int J Med Robot. 2009;5(4): 381-391.
Medical Devices: Evidence and Research

\section{Publish your work in this journal}

Medical Devices: Evidence and Research is an international, peerreviewed, open access journal that focuses on the evidence, technology, research, and expert opinion supporting the use and application of medical devices in the diagnosis, treatment and management of clinical conditions and physiological processes. The identification of novel

\section{Dovepress}

devices and optimal use of existing devices which will lead to improved clinical outcomes and more effective patient management and safety is a key feature. The manuscript management system is completely online and includes a quick and fair peer-review system. Visit http://www. dovepress.com/testimonials.php to read real quotes from authors. 